\title{
MASSIVE ORBITAL METASTASIS OF HEPATOCELLULAR CARCINOMA.
}

\begin{abstract}
Marcelo C. Machado-Netto, Emílio C. A. Lacerda, Thaís Heinke, Danielle C. C. Maia, Márcia S. Lowen and Everardo D. Saad
\end{abstract}

Compared to other organs, the eyes are relatively infrequent sites of metastasis. In a landmark case series, Ferry and Font described 227 cases of carcinoma metastatic to the eyes. ${ }^{1,2}$ Among these cases, only 28 (12\%) were orbital metastases, most frequently from tumors of the breast, lung, and genitourinary tract; in this series, the diagnosis of orbital metastasis preceded the diagnosis of the primary tumor in $61 \%$ of cases. $^{2}$ Metastases represent from $1 \%$ to $13 \%$ of orbital tumors, the rest being accounted for by primary tumors. We present the case of a patient in whom orbital metastasis was the first manifestation of cancer.

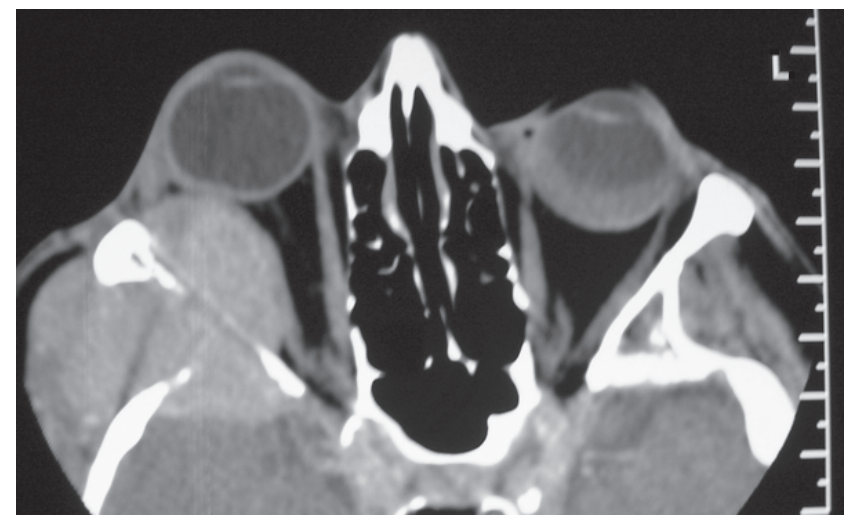

Figure 1. Computed tomography at the level of the eyes shows an orbital soft tissue mass leading to bone erosion and intracranial invasion.

\section{CASE REPORT}

A 57-year-old man presented with a 6-month history of orbital swelling in December 2003. He complained of progressive bulging of the temporal aspect of his right orbit, but postponed his medical visit for approximately 6 months. On presentation, the patient had a temporal nodule, proptosis, and mild visual discomfort, but was otherwise asymptomatic. A computed tomography (CT) scan showed an orbital soft tissue mass leading to bone erosion and intracra-

Discipline of Gastroenterology, the Multidisciplinary Oncology Group and the Department of Pathology, Federal University of Sao Paulo, Sao Paulo, Brazil.

Email: evesaad@yahoo.com

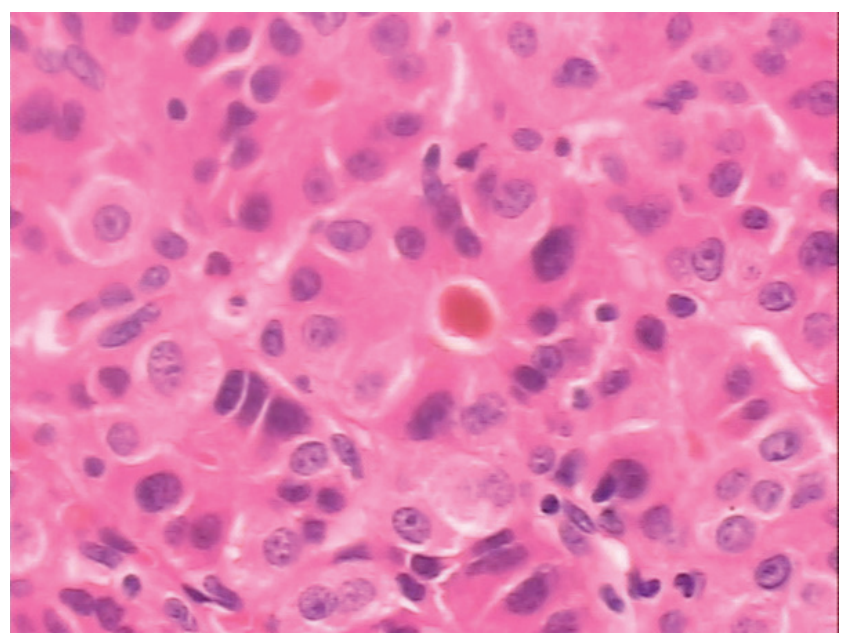

Figure 2, Panel A. Biopsy of orbital mass shows a neoplasm with pseudoglandular, trabecular pattern of polygonal cells, prominent nucleoli, vesicular nuclei, and bile plugs (hematoxylin and eosin staining; magnification, 400x).

nial invasion (Figure 1). Percutaneous incisional biopsy of the lesion showed a neoplasm with a pseudoglandular, trabecular pattern of polygonal cells, prominent nucleoli, vesicular nuclei, and bile plugs, consistent with hepatocellular carcinoma (Figure 2, Panel A). On immunohistochemistry, the tumor cells stained positive for a low-molecularweight cytokeratin (CAM 5.2), and carcinoembryonic antigen (CEA) staining outlined the bile canaliculi (Figure 2, Panels B and C). A CT scan of the liver showed a focal perfusion abnormality in the left lobe (Figure 3, arrow). The serum level of alpha-fetoprotein was normal. Hepatitis-B surface antigen was detected in the serum, and antihepatitis $\mathrm{C}$ virus antibodies were negative. The patient initially refused treatment and was lost to follow up for several months. He presented again in July 2004, with massive growth of the orbital tumor since diagnosis (Figure 4). He had progressive visual loss, but no significant pain. Given the impossibility of effective surgical or radiotherapeutic palliation and the progressive destruction of adjacent tissues, he received systemic treatment with megestrol acetate and gemcitabine. One month after treatment initiation, he 


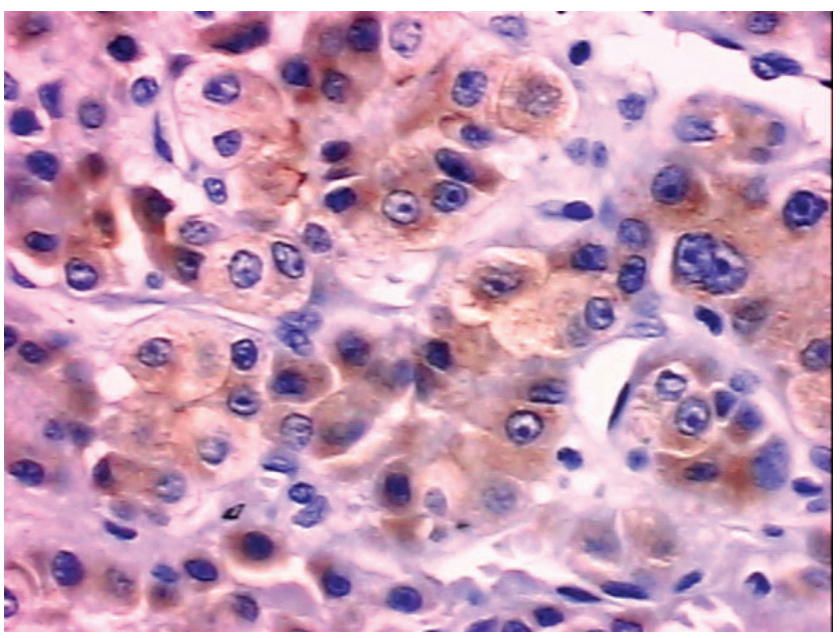

Figure 2, Panel B. Immunohistochemistry shows positive staining of the tumor cells with a low-molecular-weight cytokeratin, CAM 5.2 (magnification, 400x).

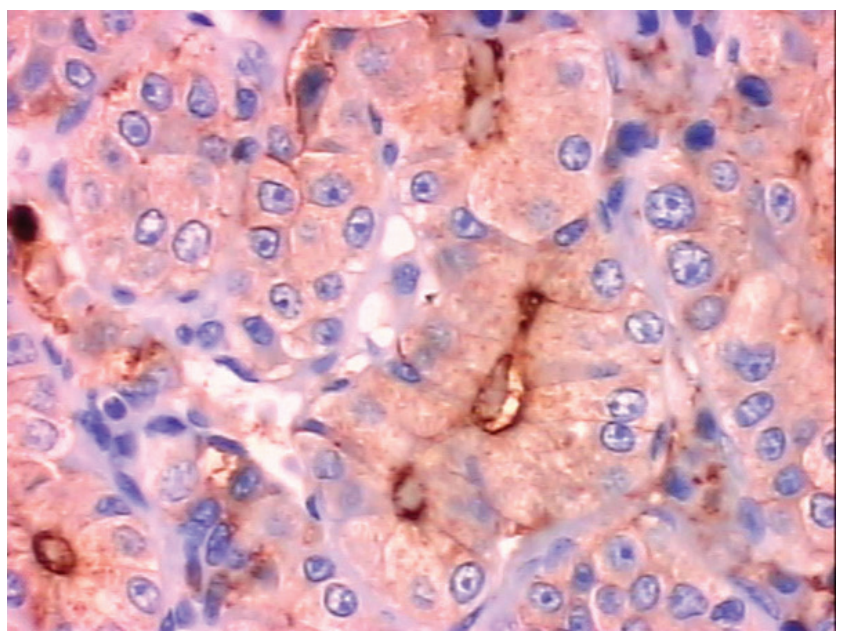

Figure 2, Panel C. Immunohistochemistry shows positive staining of the tumor cells and bile canaliculi with carcinoembryonic antigen (magnification, 400x).

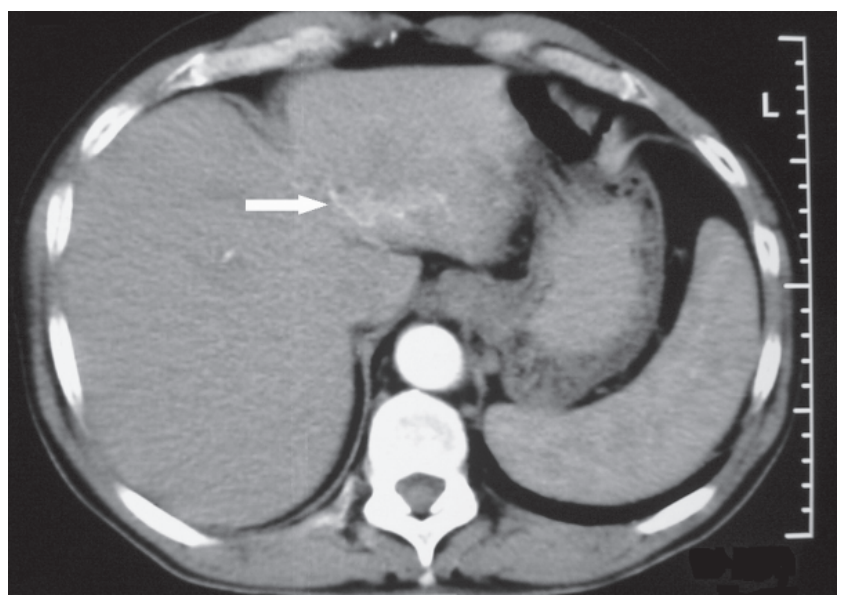

Figure 3. Computed tomography of the abdomen shows a focal perfusion abnormality in the left lobe of the liver (arrow).

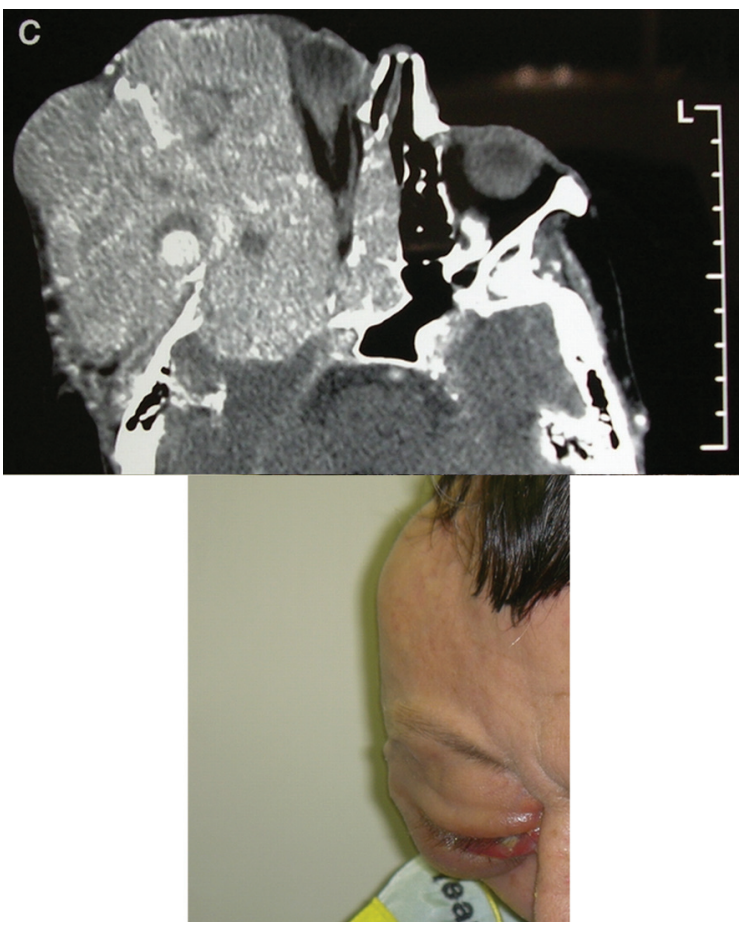

Figure 4. Massive growth of the orbital mass is shown on computed tomography (top) and clinically (bottom).

reported symptomatic improvement and had an increased performance status, but had no objective tumor response. Chemotherapy was discontinued after 4 cycles. He eventually experienced progression of the orbital lesion, and died in March 2005, 15 months after initial presentation.

\section{DISCUSSION}

Orbital metastasis is a clinically infrequent phenomenon, accounting for approximately 1 in 8 of the tumors that metastasize to the ocular structures. ${ }^{1}$ In some of the largest Western series reported to date, the most common primary tumors leading to orbital metastases were breast, lung, and prostate cancers. In most of these series, no cases of hepatocellular carcinoma were described. In contrast, a recent review from Japan has shown that hepatocellular carcinoma is the third most frequent cause of orbital metastasis in that country, after lung and breast cancer. ${ }^{4}$ In Japan, hepatocellular carcinoma is among the leading causes of cancer incidence and death. Although liver cancer is a major health problem in Japan, China, South East Asia, and sub-Saharan Africa, Brazil is a country with an intermediate age-standardized incidence rate ${ }^{5}$ and in which the disease is not among the 10 most frequent tumor types. ${ }^{6}$

Other case reports of hepatocellular carcinoma metastatic to the orbit have appeared in the Western literature in the past 2 decades and have been reviewed more recently. ${ }^{7}$ Most of these cases were reported in ophthalmol- 
ogy journals of the US and Europe. As for our patient, in many of these case reports, the orbital metastasis was often the first manifestation of hepatocellular carcinoma. ${ }^{8,9}$ Likewise, in the Japanese series of 18 cases, approximately $80 \%$ of the patients had orbital metastasis as the first sign of liver cancer. ${ }^{4}$ It is not always clear whether clinically apparent orbital metastases from hepatocellular carcinoma represent local extension from an osseous lesion or metastatic deposits in the soft tissues of the orbit. ${ }^{4}$ Proptosis, visual disturbance, and ocular pain are the most frequent clinical findings. ${ }^{4,7}$ There seems to be no lateral preponderance for orbital involvement by hepatocellular carcinoma, but the lesion was consistently unilateral in the case reports and series.

The pathological diagnosis of an orbital metastasis from hepatocellular carcinoma is usually straightforward and commonly guides the investigation toward the primary tumor. The typical appearance is that of a trabecular pattern associated with polygonal cells that have an acidophilic cytoplasm, large round or oval nuclei, and prominent nucleoli. Immunohistochemistry may be helpful and typically demonstrates staining of tumor cells with CAM 5.2, and of bile canaliculi with polyclonal CEA. Although serum levels of alpha-fetoprotein are elevated in up to $90 \%$ of patients, ex- pression on immunohistochemistry is only found in $30 \%$ to $50 \%$ of the primary tumors. ${ }^{7}{ }^{10}$ Interestingly, other cases of orbital metastases with normal serum levels ${ }^{9}$ or negative tissue expression of alpha-fetoprotein have been reported.?

Treatment for metastatic hepatocellular carcinoma is of limited efficacy. In the cases of orbital metastases from hepatocellular carcinoma reported to date, focal radiotherapy has been the most frequent treatment modality. Although surgery has typically been limited to diagnostic purposes, an occasional patient may derive benefit from local excision. Some patients have also been treated with systemic chemotherapy. Currently, no chemotherapeutic agent may be considered standard, and hormonal agents such as megestrol acetate and tamoxifen may provide palliative benefit to some patients. In our patient, symptomatic improvement followed the administration of megestrol acetate and gemcitabine, but we were uncertain as to the relative contribution of these agents. The average survival of patients with orbital metastases from hepatocellular carcinoma has been reported as 10.4 months, ${ }^{4}$ and often depends on the disease status elsewhere in the body. Our report illustrates the possibility of massive growth of an untreated orbital metastasis of hepatocellular carcinoma, and reminds clinicians of this possible cause of orbital tumor.

\section{REFERENCES}

1. Ferry AP, Font RL. Carcinoma metastatic to the eye and orbit. I. A clinicopathologic study of 227 cases. Arch Ophthalmol. 1974;92:27686.

2. Font RL, Ferry AP. Carcinoma metastatic to the eye and orbit. III. A clinicopathologic study of 28 cases metastatic to the orbit. Cancer. 1976;38:1326-35.
3. Shields JA, Shields CL, Brotman HK, Carvalho C, Perez N, Eagle RC, Jr. Cancer metastatic to the orbit: The 2000 Robert M. Curts Lecture. Ophthal Plast Reconstr Surg. 2001;17:346-54.

4. Amemiya T, Hayashida H, Dake Y. Metastatic orbital tumors in Japan: a review of the literature. Ophthalmic Epidemiol. 2002;9:35-47. 
5. Stewart BW, Kleihues P (editors). World Cancer Report. Lyon: IARC Press; 2003.

6. Brasil. Ministério da Saúde. Instituto Nacional de Câncer. Estimativa 2005: Incidência de Câncer no Brasil. Disponível em http:// www.inca.gov.br/estimativa/2005/.

7. Font RL, Maturi RK, Small RG, Garcia-Rojas M. Hepatocellular carcinoma metastatic to the orbit. Arch Ophthalmol. 1998;116:942-9.
8. Zubler MA, Rivera R, Lane M. Hepatoma presenting as a retro-orbital metastasis. Cancer. 1981;48:1883-5.

9. Barbat V, Morin Y, Metge F, Hamard H. Carcinome hépatocellulaire: un cas de métastase orbitaire révélatrice. Rev Med Interne. 2000;21:8690 .

10. Torbenson M, Kannangai R, Abraham S, Sahin F, Choti M, Wang J. Concurrent evaluation of $\mathrm{p} 53$, beta-catenin, and alpha-fetoprotein expression in human hepatocellular carcinoma. Am J Clin Pathol. $2004 ; 122: 377-82$ 